\title{
Performance Evaluation of IEEE 802.15.4 OQPSK and CSS PHY in the Presence of Interference
}

\author{
Felix Wunsch, Holger Jäkel, Friedrich K. Jondral \\ Communications Engineering Lab (CEL) \\ Karlsruhe Institute of Technology (KIT) \\ Karlsruhe, Germany \\ Email: $\{$ felix.wunsch, holger.jaekel, friedrich.jondral\}@kit.edu
}

\begin{abstract}
Industrial environments often exhibit harsh propagation conditions for wireless transmissions. This paper investigates the influence of different interference characteristics on the performance of IEEE 802.15.4 using the OQPSK and Chirp Spread Spectrum (CSS) PHY. Bit error rates are determined for AWGN, Rayleigh fading and self-interference scenarios. GNU Radio, a Software Defined Radio framework, serves as simulation environment. In addition to the implementation of the CSS transmitter and receiver, a low-complexity synchronization algorithm, which only consists of a correlator and a second order Phase Locked Loop (PLL), and its limitations are presented. Furthermore, MAC layer simulations are performed for beaconenabled (slotted) as well as nonbeacon-enabled (unslotted) mode to make a statement about achievable throughput and latency in the presence of interference. It shows that the CSS PHY clearly surpasses the OQPSK PHY in throughput. Moreover, the simulation results are confirmed by the derivation of the theoretical maximum throughput for the investigated PHY layers.
\end{abstract}

Keywords-CSS, IEEE 802.15.4, Interference Analysis, OQPSK, Throughput, Wireless Sensor Networks (WSNs)

\section{INTRODUCTION}

Smart Factories and the Internet of Things are currently thriving research topics that have the potential to radically change the way production processes work. Where today largescale mass production is necessary to reduce cost, the factory of the future might be able to work with small-scale series and per-product configurability. This is enabled by so-called Smart Products that carry all information needed for their fabrication, e.g., on RFID tags. A large number of sensors and actuators communicate with the product and flexibly guide it through the manufacturing process. The available information can be used to optimize stock-keeping, thus resulting in Smart Logistics, another aspect of the Smart Factory of tomorrow.

Wired connection of all the devices in such a factory can be costly, time-consuming and inflexible. This can be alleviated by employing wireless technologies and so called Low Range Wireless Personal Area Networks (LR-WPANs), creating Wireless Sensor Networks (WSNs). Wireless sensor nodes are inexpensive, simple to set up (even on mobile objects) and can be self-configuring. Battery lifetime is easily in the order of years, thus reducing maintenance cost. Using ISM bands for communication also avoids the need for expensive spectrum licenses. Cost and energy efficiency are two of the main design goals in the design of new devices.

Reliability and latency requirements are key concerns for WSNs, e.g. when an emergency alert has to be transmitted, and can therefore be very strict. Wireless technology generally struggles with this kind of requirements due to the unpredictability of the transmission medium. To address these problems, several institutions have developed transmission standards, such as WirelessHART, ISA 100.11a, and ZigBee. All these documents have in common that they use or are at least based on IEEE 802.15.4. The standard features multiple PHY layers, two of them being the widely used OQPSK and the more recent Chirp Spread Spectrum (CSS) PHY that are investigated in this paper. To achieve a fair comparison between the two PHY layers, Bit Error Rate (BER) measurements are performed in various interference scenarios.

Simulations are performed in GNU Radio [1], a free and open source Software Defined Radio (SDR) framework. It allows modular and block-based signal-processing in $\mathrm{C}++$ or Python with easy access to RF hardware. Based on the existing gr-ieee802-15-4 library, the CSS PHY is implemented and added [2]. There is also a low-complexity synchronization algorithm for CSS included, thus allowing over-the-air transmissions.

With the results from the previous simulations, the MAC layer (in both beacon-enabled (slotted) and nonbeacon-enabled (unslotted) mode) is investigated in terms of throughput and latency for different channel occupation probabilities and bit error rates.

The remainder of the paper is organized as follows: Section II discusses previous works relevant for this paper. An overview of the IEEE 802.15.4 standard is given in section III. The implementation of PHY and MAC layer as well as the simulation setup are presented in section IV with the results given in section V. Section VI contains the concluding remarks.

\section{RELATED WORKS}

The performance of the beacon-enabled MAC protocol is investigated e.g. in the works of Pollin et al. [3], Koubaa et al. [4], Buratti [5], and Park et al. [6].

An analytical evaluation of the nonbeacon-enabled MAC is given in Buratti's and Verdone's article [7]. Chowdhury et al. [8] also present performance results for the nonbeacon-enabled MAC explicitly considering the CSS PHY.

All mentioned works have in common that they do not investigate the impact of different bit error ratios on the overall throughput and delay. 
Binary Data
From PPDU $\rightarrow$ Bit-to-symbol $\rightarrow$ Symbol-to-chip $\rightarrow$ OQPSK modulator $\rightarrow$ Modulated signal

(a) OQPSK PHY modulator

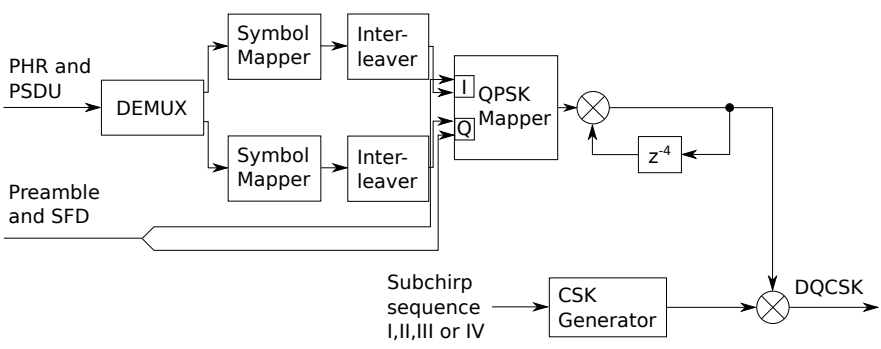

(b) CSS PHY modulator

Fig. 1. IEEE 802.15.4 PHY modulators [9]

To the best of the authors' knowledge, this article also is the first analysis of the interference resilience of the CSS PHY.

\section{IEEE 802.15.4 OVERVIEW}

The IEEE 802.15.4 standard defines a MAC layer and multiple PHY layers, of which the OQPSK and the CSS PHY are discussed in the scope of this article.

\section{A. PHY Layer}

The PHY Protocol Data Unit (PPDU) consists of a preamble and a Start-of-frame Delimiter (SFD) used for synchronization, followed by the PHY Header (PHR) which indicates the length of the following payload, the PHY Service Data Unit (PSDU).

The OQPSK PHY is actually a Direct Sequence Spread Spectrum (DSSS) system with OQPSK modulated chips. Incoming bits are grouped and mapped to PN sequences, which are then OQPSK modulated. The modulator block diagram is depicted in Fig. 1(a).

As it can be seen in Fig. 1(b), modulating a CSS PHY signal is considerably more complex. After grouping the bits and mapping them to chip sequences in a similar way as in OQPSK PHY, the chips are DQPSK encoded. As two different data rates are supported $(250 \mathrm{kbit} / \mathrm{s}$ and $1 \mathrm{Mbit} / \mathrm{s})$, this choice controls the bit to chip encoding rate. An additional block interleaver is employed if the slower data rate is desired. The distinctive feature, from which CSS derives its name, is the Chirp Sequence Keying (CSK), generating chirp sequences consisting of four subchirps. There are four different chirp sequences that can be used to separate users due to their low cross-correlation. Every subchirp is a frequency ramp with either decreasing or increasing frequency in the upper or lower sideband of the channel. Every subchirp is weighted with one DQPSK symbol, resulting in a modulation named Differential Quadrature Chirp Shift Keying (DQCSK).

\section{B. MAC Layer}

The standard offers two different modes to orchestrate the channel access, beacon-enabled and nonbeacon-enabled mode. Both modes allow the optional request of an acknowledgment frame.

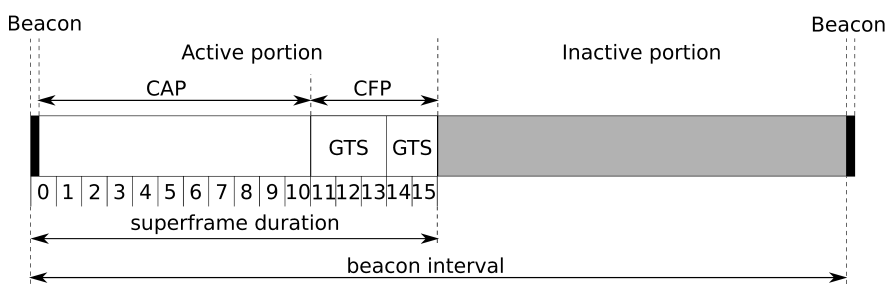

Fig. 2. Structure of the MAC superframe in beacon-enabled mode [9]

In Nonbeacon-enabled mode, there is no synchronization among the devices and a simple Carrier Sense Multiple Access with Collision Avoidance (CSMA-CA) scheme is used.

Beacon-enabled mode provides a common time base for all devices in the network by creating superframes. They consist of an active and an inactive period. Beginning with the transmission of a beacon frame, the active portion, which is divided into equally spaced time slots, consists of a Contention Access Period (CAP) and a Contention Free Period (CFP). During the CAP, a slotted version of the CSMA-CA algorithm is used. Time slots in the CFP can be requested from the coordinator of the network. For this paper, the CAP is assumed to span the whole active period.

If energy-efficiency is a concern, beacon-enabled mode has a significant advantage. Active and inactive periods can be adjusted independently from the order of milliseconds to minutes, thus adapting to the individual communication needs of a system. During the inactive period, the transceiver can enter a low-power sleep state and therefore prolong battery lifetime. Also, usage of the CFP enables transmissions with a bounded latency, which is not possible in nonbeacon-enabled mode.

\section{IMPLEMENTATION}

\section{A. CSS PHY}

The gr-ieee802-15-4 project is a GNU Radio library based on the work of Thomas Schmid at the University of California, Los Angeles (UCLA). It already implements the OQPSK PHY for the $2.4 \mathrm{GHz}$ ISM band. Continuing the development, code for the CSS PHY is added, including a complete transceiver. In this context, a low-complexity synchronization algorithm is proposed. Due to complexity constraints, the implementation assumes the chosen data rate mode and the packet length to be constant during runtime. The transmitter implementation generally follows the reference modulator structure as in Fig. 1(b). The receiver consists of a soft decision demodulator and a synchronization part, which shall be discussed in this section.

\section{B. Synchronization Algorithm for CSS PHY}

Due to the properties of the CSS signal, only a correlator for chirp detection and a second order Phase Locked Loop (PLL) are required to obtain time, phase and frequency synchronization. The chirp detector calculates the correlation coefficient $\rho$ between the received signal $s$ and the soughtafter chirp sequence $r$ as in (1). The simplification is justified because the signals are assumed to be zero-mean. If the result exceeds a certain threshold, it is treated as valid DQPSK symbol, otherwise it is discarded. 


$$
\rho=\frac{\sum_{i=0}^{N-1} s_{i} \cdot r_{i}^{*}}{\sqrt{\sum_{i=0}^{N-1}\left|s_{i}\right|^{2} \cdot \sum_{i=0}^{N-1}\left|r_{i}\right|^{2}}}
$$

The DQPSK symbols are then fed into the PLL which applies the following algorithm:

1) Correct input symbol by previous phase offset

2) Find minimum angular distance to the nearest constellation point and correct a second time by the remaining offset, return the symbol

3) Increase the stored phase offset from the previous run by the new offset found in this iteration

Of course, the ability to correct frequency offsets is limited. The phase shift $\phi_{o}$ caused by the frequency offset $f_{o}$ during one symbol period must be smaller than the angle between any constellation point and the nearest decision boundary. In this example, this corresponds to $\pi / 4$ or $45^{\circ}$, thus yielding

$$
\phi_{o}=2 \pi f_{o} t_{\text {sym }} \stackrel{!}{<} \pi / 4 .
$$

Solving (2) for $f_{o}$ leads to

$$
f_{o}<\frac{1}{8 \cdot t_{\mathrm{sym}}}
$$

With a maximal symbol time of $t_{\mathrm{sym}}=3.1875 \mu \mathrm{s}$ as given in [9], inserting into (3) yields a maximal correctable frequency offset of about $39.2 \mathrm{kHz}$. The proper functionality of the implementation has been verified in an over-the-air test.

\section{C. $M A C$}

The IEEE 802.15.4 MAC algorithms are implemented in pure Python. It would be desirable to have them in GNU Radio as well, but latencies caused by the non-deterministic OS behavior and communication with RF devices through Ethernet or USB violate the timing-constraints of the standard.

Both beacon-enabled and nonbeacon-enabled mode are implemented. As the exact timings depend on the underlying PHY layer, that and many other parameters are configurable, the most important ones being the bit error ratio, the packet collision probability and the frame length.

\section{Simulation}

All PHY layer simulations are carried out with modified GNU Radio scripts by creating the flow graphs in GNU Radio Companion and then manually adding the BER measurement logic.

Even though synchronization algorithms are essential for the practical usage of any wireless system, perfect synchronization is assumed to avoid comparing the performance of synchronization algorithms instead of the inherent robustness of the modulation schemes.

For the conversion from SNR to $E_{b} / N_{0}$, the following formula is used:

$$
\frac{E_{b}}{N_{0}}=\frac{S}{N} \cdot \frac{B}{R}, \quad B: \text { bandwidth }[\mathrm{Hz}], R: \text { bit rate }[\mathrm{bit} / \mathrm{s}]
$$

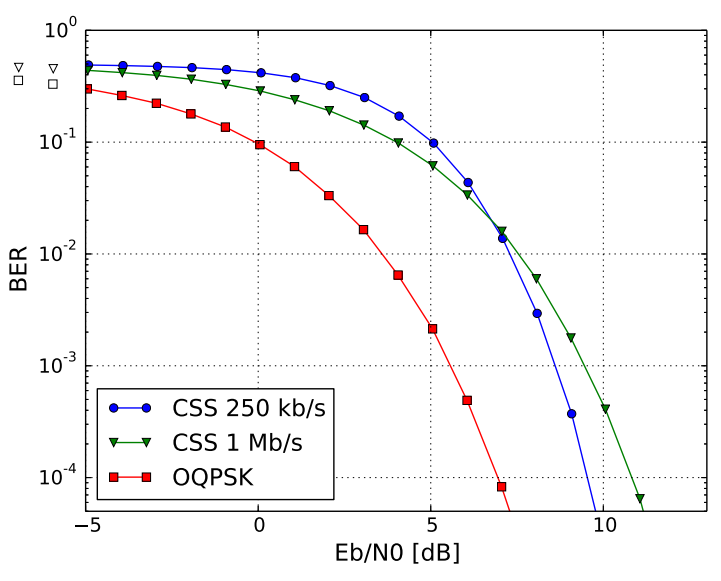

Fig. 3. BER performance in AWGN channel

Obviously, the conversion heavily depends on the definition of the bandwidth. This paper uses the baseband sample rate as bandwidth, i.e., $4 \mathrm{MHz}$ for OQPSK and $32 \mathrm{MHz}$ for CSS PHY, respectively. Using the $20 \mathrm{~dB}$ bandwidth instead "improves" the CSS curves by almost $3 \mathrm{~dB}$. This should be kept in mind when comparing the performance of both modulation schemes.

\section{RESUlts}

\section{A. $A W G N$}

Probably the most common but still insightful scenario is AWGN. The signal model is

$$
s_{r}(t)=s_{t}(t)+w(t)
$$

with $s_{r}(t)$ the received signal, $s_{t}(t)$ the transmitted signal and $w(t)$ the noise. The outcome of the simulation is depicted in Fig. 3. Its results for the OQPSK PHY also coincide with the work of Mantri et al. [10].

It appears that the higher spreading factor of the CSS PHY results in a considerably steeper slope than it is the case for the OQPSK PHY.

\section{B. Rayleigh Fading}

More realistic scenarios include for example factory halls with harsh transmission conditions. In those environments, the reception quality can suffer from a missing line-of-sight connection and the interference of multipaths. The signal model becomes

$$
s_{r}(t)=h(\tau, t) * s_{t}(t)+w(t)
$$

with $h(\tau, t)$ representing the time-varying channel impulse response. The distribution of the signal power along the time axis is called Power Delay Profile (PDP). Its values represent the mean power of the respective multipath components which are zero-mean and Gaussian distributed.

In the simulation, a channel with only 2-tap PDP, i.e., two multipath components, is chosen. The taps are located at $h(0)$ (due to perfect time synchronization) and $h(250 \mathrm{~ns})$ with values of 0.91 and 0.09 , respectively. This corresponds to a coherence bandwidth of about $4 \mathrm{MHz}$ and a relative velocity of $25 \mathrm{~m} / \mathrm{s}$. The structure of the PDP is based on the time 


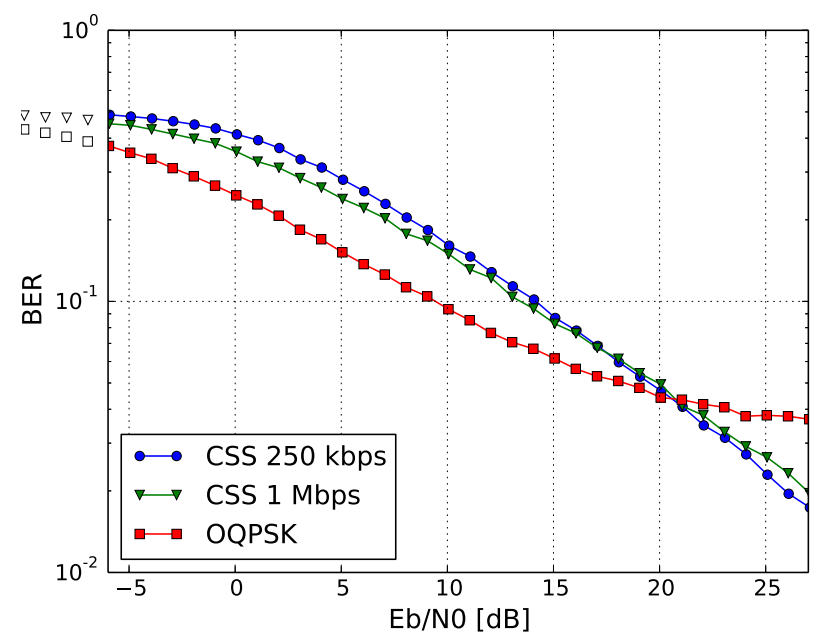

Fig. 4. BER performance in 2-tap Rayleigh channel

resolution offered by the OQPSK PHY, which is $250 \mathrm{~ns}$, and the restriction to a maximum propagation path length of $75 \mathrm{~m}$, a reasonable assumption for industrial environments.

Fig. 4 shows a clear difference in the error characteristic between OQPSK and CSS PHY. While the CSS curve declines gradually, OQPSK runs into an error floor where the BER does not decrease any further with increasing signal power. This is the point where CSS shows its strengths, originally being designed for mobile scenarios.

\section{CSS Self-Interference}

Featuring four different, almost-orthogonal chirp sequences, the CSS PHY allows the parallel use of the same channel by up to four devices. This yields the following signal model:

$$
s_{r}(t)=\sum_{m=0}^{M-1} s_{m}(t)+w(t)
$$

with $m$ being the chirp sequence index and $s_{m}(t)$ being the different users' transmit signals. They are assumed to be perfectly synchronous with equal power level. If there are no other users (or in this case, interferers), this is equal to the simple AWGN scenario discussed in Section V-A.

Looking at the results depicted in Fig. 5 it is striking that the performance degrades considerably with the addition of the second interferer, while the first parallel user only marginally impacts the BER. The reason for this behavior is to be found in the specific design of the chirp sequences as described in Section III-A. The chirp sequences of user 2 and 4 are using the opposing sidebands for their subchirps whereas user 3 shares the same sideband with frequency ramps going the opposite way. Obviously, the frequency separation through multiple sidebands achieves a lower cross-correlation than the usage of different subchirps.

\section{Throughput and Latency}

The simulations in this chapter combined with the results of the previous chapters allow a direct mapping from $E_{b} / N_{0}$ values in a specific interference scenario to achievable throughput

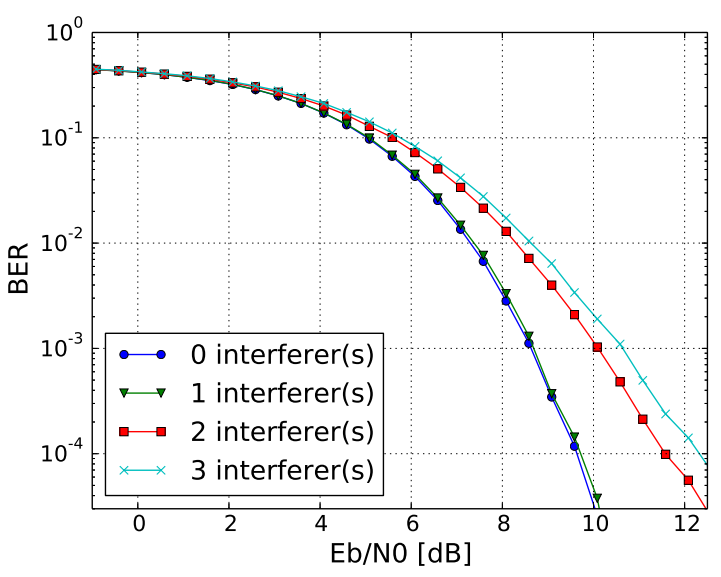

(a) $250 \mathrm{kbit} / \mathrm{s}$ mode

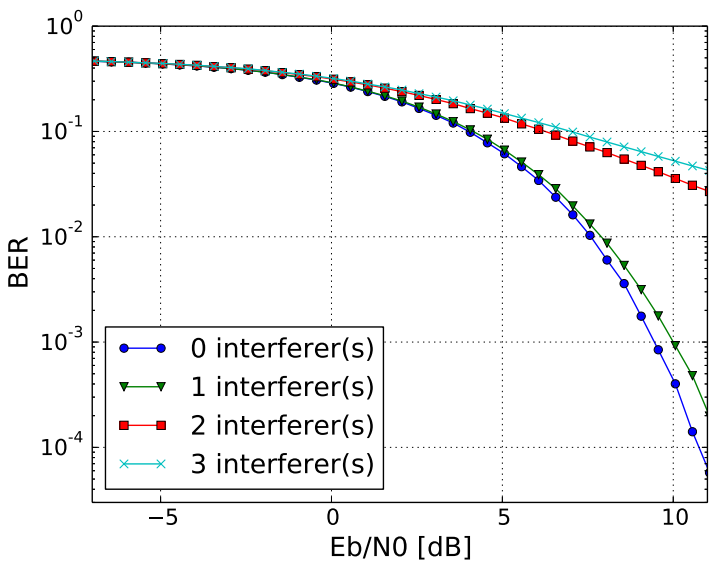

(b) $1 \mathrm{Mbit} / \mathrm{s}$ mode

Fig. 5. CSS PHY self-interference

and latency at the interface between the MAC and upper layers, i.e., the throughput measured in this section refers to the MAC payload. A transmission is considered to be successful, when the data frame and its acknowledgment frame are received and the CRC check does not indicate any bit errors.

Depicted in Fig. 6 are the results for the nonbeaconenabled MAC. Simulations for beacon-enabled MAC are also performed and show a decrease of about $20 \%$ in throughput and also a slight increase in latency due to the more complex medium access scheme. This is tested for a configuration with minimal overhead compared to the unslotted algorithm.

The maximum throughput in a free channel with no transmission errors can also be calculated as

$$
R_{\max }=\frac{\text { MAC payload per frame }}{\text { Transmission time per frame }} .
$$

Considering a minimum length MAC data frame header (including the CRC), 120 byte remain as MAC payload. The timespan needed for the transmission of this frame is composed of the average CSMA-CA backoff time, the duration of the data frame, inter-frame spacings and the duration of the acknowledgment frame. Table I shows the results for the different PHY layers.

Due to the PHY-dependent timings in the MAC protocol, the CSS PHY achieves a higher throughput than OQPSK at the 
TABLE I. MAXIMUM THROUGHPUT IN NONBEACON-ENABLED MODE

\begin{tabular}{|l|l|l|l|}
\hline PHY & OQPSK & CSS 250 kb/s & CSS 1 Mb/s \\
\hline Frame transmission time [ms] & 5.312 & 4.844 & 1.443 \\
\hline Max. data rate [kb/s] & 149.25 & 182.36 & 515.34 \\
\hline
\end{tabular}

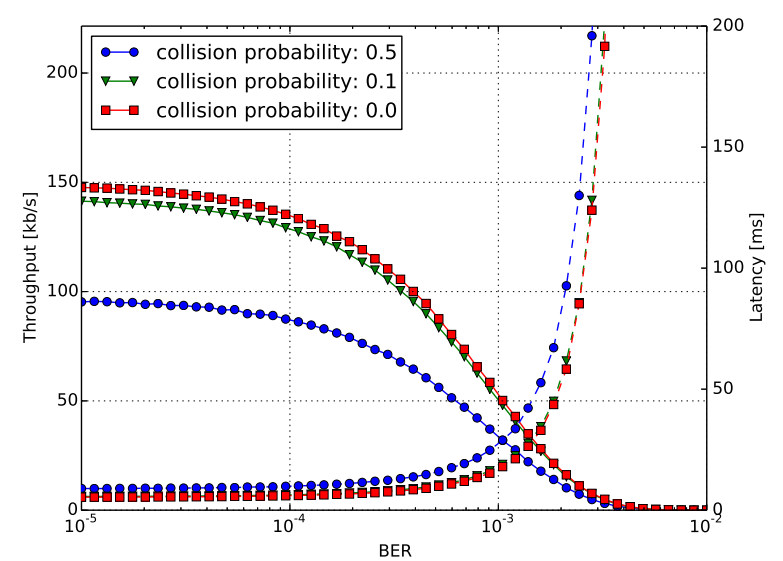

(a) OQPSK PHY

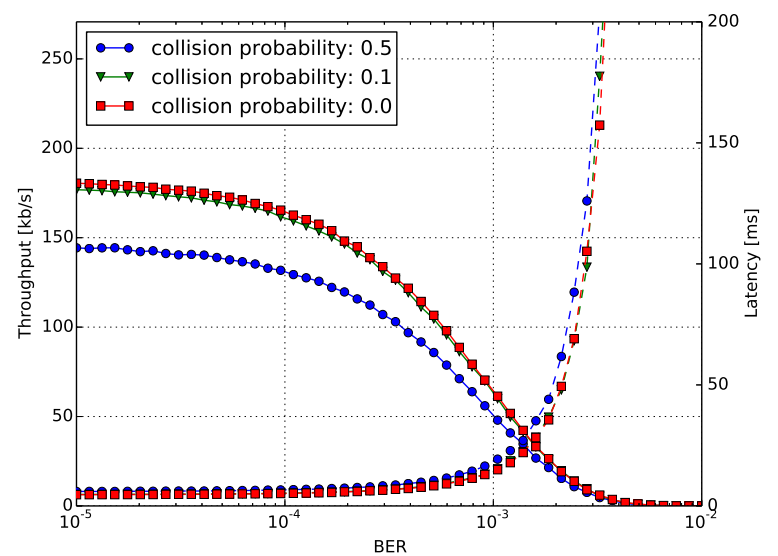

(b) CSS PHY in $250 \mathrm{kbit} / \mathrm{s}$ mode

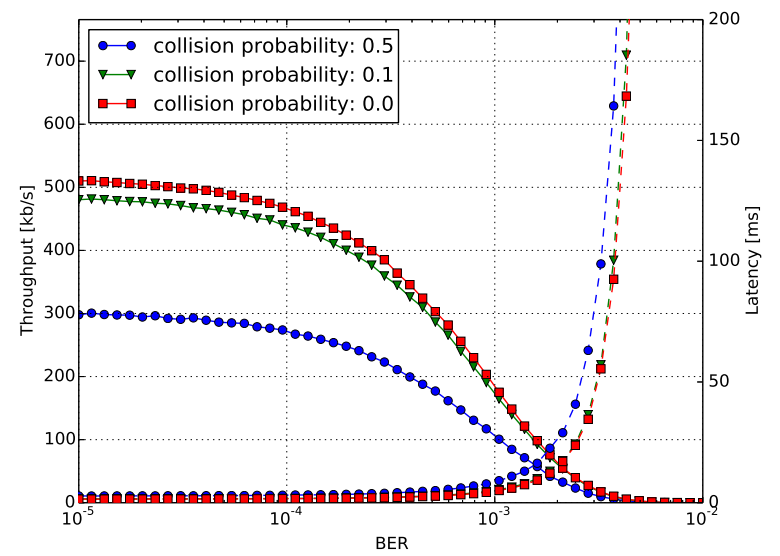

(c) CSS PHY in $1 \mathrm{Mbit} / \mathrm{s}$ mode

Fig. 6. IEEE 802.15.4 nonbeacon-enabled MAC performance with maximum length frames

same gross data rate of $250 \mathrm{kbit} / \mathrm{s}$. It also recovers faster from a denied channel access due to shorter backoff periods, leading to an optimized throughput even in heavily loaded channels.

\section{CONCLUSION}

The IEEE 802.15.4 implementation for GNU Radio which is presented in this paper features a complete transceiver employing the CSS PHY. The proposed low-complexity synchronization algorithm can correct and track frequency offsets of up to $39.2 \mathrm{kHz}$.

The simulations show that the BER performance of OQPSK and CSS PHY appears to be relatively equal in most scenarios. Even when CSS performs better, this is bought with an increased computational effort due to the considerably more complex modulator structure and higher baseband sample rate. However, when comparing OQPSK and CSS PHY in conjunction with the MAC layer, CSS turns out clearly ahead due to shorter timings. Its net throughput (in nonbeaconenabled mode) of $182.36 \mathrm{kbit} / \mathrm{s}$ at the interface between MAC and upper layers is about $20 \%$ higher although both PHY layers support the same gross PHY data rate of $250 \mathrm{kbit} / \mathrm{s}$. Switching to beacon-enabled mode reduces the throughput by about $20 \%$ due to the more complex MAC protocol.

The combination of the PHY and MAC layer simulation results allow a direct mapping from interference scenarios with a certain $E_{b} / N_{0}$ ratio to the expected throughput and latency.

In the near future, more over-the-air measurements will be carried out to assess the performance of the CSS PHY with the proposed synchronization algorithm under real-world conditions.

\section{REFERENCES}

[1] “Official GNU Radio website," http://www.gnuradio.org, Accessed: 2015-02-17.

[2] "Github repository for gr-ieee802-15-4," https://github.com/fewu/grieee802-15-4, Accessed: 2015-02-17.

[3] S. Pollin, M. Ergen, S. Ergen, B. Bougard, L. Der Perre, I. Moerman, A. Bahai, P. Varaiya, and F. Catthoor, "Performance Analysis of Slotted Carrier Sense IEEE 802.15.4 Medium Access Layer," Wireless Communications, IEEE Transactions on, vol. 7, no. 9, pp. 3359-3371, September 2008.

[4] A. Koubaa, M. Alves, and E. Tovar, "A comprehensive simulation study of slotted CSMA/CA for IEEE 802.15.4 wireless sensor networks," in Factory Communication Systems, 2006 IEEE International Workshop on, 2006, pp. 183-192.

[5] C. Buratti, "Performance analysis of ieee 802.15 .4 beacon-enabled mode," Vehicular Technology, IEEE Transactions on, vol. 59, no. 4, pp. 2031-2045, May 2010.

[6] T. Park, T. Kim, J. Choi, S. Choi, and W. Kwon, "Throughput and energy consumption analysis of IEEE 802.15.4 slotted CSMA/CA," Electronics Letters, vol. 41, no. 18, pp. 1017-1019, Sept 2005.

[7] C. Buratti and R. Verdone, "Performance Analysis of IEEE 802.15.4 Non Beacon-Enabled Mode," Vehicular Technology, IEEE Transactions on, vol. 58, no. 7, pp. 3480-3493, Sept 2009.

[8] M. Chowdhury, N. Ullah, M. Kabir, P. Khan, and K. S. Kwak, "Throughput, delay and bandwidth efficiency of IEEE 802.15.4a using CSS PHY," in Information and Communication Technology Convergence (ICTC), 2010 International Conference on, Nov 2010, pp. 158163.

[9] "IEEE Standard for Local and metropolitan area networks-Part 15.4: Low-Rate Wireless Personal Area Networks (LR-WPANs)," IEEE Std 802.15.4-2011 (Revision of IEEE Std 802.15.4-2006), pp. 1-314, Sept 2011.

[10] M. Mantri, P. Velagapudi, B. Eravatri, and V. Mani, "Performance analysis of 2.4GHz IEEE 802.15.4 PHY under various fading channels," in Emerging Trends in Communication, Control, Signal Processing Computing Applications (C2SPCA), 2013 International Conference on, Oct 2013, pp. 1-4. 